\title{
Parametric study of the influence of the structure dimensions and the material properties on the global behavior of plates made of composite materials
}

\author{
Mohamad Abdul Wahab ${ }^{1}$, Nazih Moubayed ${ }^{1, a}$, Tony Jabbour ${ }^{2}$ and Peter Davies ${ }^{3}$ \\ 1 Faculty of Engineering 1, Lebanese University, Tripoli, Lebanon \\ 2 ISAE, Lebanese University, Beirut, Lebanon \\ 3 Laboratory of Materials of Marins, Center of Brest, BP 70, 29280 Plouzané, France
}

Received 25 May 2012, Accepted 28 May 2013

\begin{abstract}
This paper considers the effect of the damage caused by transverse stresses due to a low velocity impact loading on composite plates. The dynamic response of a plate is calculated using the modal superposition technique based on the classical plate theory of sandwich plates (stratified) taking into account transverse shear effects. An indentation law, based on the Hertz theory and verified experimentally, governs the behavior of the plate subsequent to impact. The numerical time integration scheme is used to calculate the contact force at any instant by combining the indentation and the dynamic response of the structure. The obtained results show a good correlation between the shock results and their prediction. This method is equally applied to the contact force as well as to the deformation of the plate. The simulation permits a parametric study of the structure dimensions and the material properties on the global behavior of the plate.
\end{abstract}

Key words: Composite material / impact / dynamic response / sandwich plate / deformation

\section{Introduction}

It has been observed in sandwiched composite panels, intended for use in naval or off-shore construction, that, when those panels are subjected to shock loading, two types of damage need to be considered: a local damage involving only localized micromechanical effects inherent in the material, and a global damage evidenced by fracture cracks due to flexure on the upper and lower surfaces of the plate, or a local degradation due to shear failure in the matrix further away from the point of impact.

In order to study these types of damage that involve the whole lay-up of the structure and the boundary conditions, a dynamical model of the panel is given. This one is subjected to a shock loading which do not cause any global damage. This model needs to treat the structural aspect of the panel and its behavior during a localized damage under the impactor.

The dynamical response is obtained by modal superposition, the modes being calculated analytically assuming a simple mathematical model of the sandwiched plate vibrating under simple limiting conditions. The assumed plate model follows the Reissner-Midlin [1] and Whitney and Pagano [2] types which take into account

${ }^{a}$ Corresponding author: nmoubayed@yahoo.com the transverse shear effects. The displacement field can then be calculated at any point of the plate.

In order to calculate the dynamical loading on the structure, i.e., the contact force due to the impact, it is necessary to formulate an indentation law that would reflect the localized effects of the shock. In this paper, a law of the Hertz type has been adopted and is verified experimentally.

A numerical scheme makes it possible to integrate, with respect to time, the two mechanical phenomena, the local deformation and the response of the structure.

To validate the model, the low energy impact is achieved by dropping on the structure a rigid sphere of mass equal to $10.9 \mathrm{~kg}$ at impacting velocities ranging from 1 to $10 \mathrm{~m} \cdot \mathrm{s}^{-1}$.

The skin laminates forming the top and the bottom surfaces of the sandwiched plate are made of composite materials with continuous fibers imbedded in an organic matrix. A parametric study is then performed to study the effect of the dimensions of the plate and the mechanical properties of the material.

\section{Dynamical model}

The problem of isotropic plates and beams subjected to impact, has been considered by Timoshenko [3] while 


\section{Nomenclature}

\begin{tabular}{|lll|}
\hline Notation & Unit & Description \\
$a, b$ & $\mathrm{~m}$ & Plate dimensions \\
$B_{i j}$ & $\mathrm{~N}$ & Bending-extension coupling coefficients \\
$D_{i j}$ & $\mathrm{~N} . \mathrm{m}$ & Bending-twist coupling coefficients \\
$E_{i}, E$ & $\mathrm{~N} \cdot \mathrm{m}^{-2}$ & Young's modulus \\
$F(t)$ & $\mathrm{N}$ & Contact force \\
$F_{i}$ & $\mathrm{~N}$ & Value of force distribution within a time interval \\
$g$ & $\mathrm{~m} \cdot \mathrm{s}^{-2}$ & Acceleration \\
$G_{i j}$ & $\mathrm{~N} \cdot \mathrm{m}^{-2}$ & Shear modulus of elasticity \\
$I$ & $\mathrm{~m}^{4}$ & Moment of inertia \\
$k_{\mathrm{h}}$ & $\mathrm{N} \cdot \mathrm{m}^{-1.5}$ & Hertz coefficient \\
$L$ & & Lagrange multiplier or Lagrangian \\
$M_{m n}$ & & Generalized mode mass \\
$m_{0}$ & $\mathrm{~kg}$ & Impactor mass \\
$p(x, y)$ & $\mathrm{N} . \mathrm{m}^{-2}$ & Transverse loading \\
$q_{m n}(\mathrm{t})$ & & Unknown generalized variables \\
$Q_{m n}$ & $\mathrm{~N} . \mathrm{m}^{-2}$ & Plane stress elastic coefficients \\
$R$ & $\mathrm{~m}$ & Impactor radius \\
$g_{m n}^{3}(x, y)$ & & Deformation of the plate \\
$s(t)$ & $\mathrm{m}$ & Impactor displacement \\
$V_{d}$ & $\mathrm{~J}$ & Energy of deformation \\
$u^{\beta},(\beta=1.2)$ & $\mathrm{m}$ & Displacement of the middle surface \\
$U_{d}$ & $\mathrm{~J}$ Joule & Potential energy of the structure \\
$V_{0}$ & $\mathrm{~m} . \mathrm{s}^{-1}$ & Impactor speed before impact \\
$w$ & $\mathrm{~m}$ & Tranverse displacement of the structure \\
$x, y, z$ & $\mathrm{~m}$ & Cartesian coordinates \\
$\alpha$ & $\mathrm{m}$ & Penetration, indentation \\
$\psi_{x}, \psi_{y}$ & & Rotations of normal lines \\
$\phi_{k}$ & & Nodal vectors \\
\hline
\end{tabular}

impact loading on structures made of composite materials has been investigated by various authors [4-8]. In this paper, a simple dynamical approach has been adopted based on modal superposition. Indeed, the present study seeks to analyze the global damage modes that arise due to overloads away from the impacted regions.

\subsection{Equation of motion}

The case of a simply-supported, sandwiched, composite plate, with symmetric skin laminates, that is subjected to an impact loading, is treated. To analyze the behavior of the plate subsequent to loading, the theory developed by Whitney and Pagano [2] for multi-layered plates is utilized in the present study. This theory is based on some fundamental hypotheses which are:

- (A1) The plate is made of thin, parallel layers of constant thickness,

- (A2) Each layer behaves elastically and is under plane stress conditions,

- (A3) The deformations are small.

It should be noted that the Whitney's theory is based on an admissible kinematic field of the Reissner-Midlin type [1] which has the form:

$$
\left\{\begin{array}{l}
u_{\beta}(x, y, t)=u_{\beta}^{0}(t)+z \psi_{\beta}, \quad(\beta=x, y) \\
u_{3}(x, y, t)=w(x, y, t)
\end{array}\right.
$$

where $u_{\beta}^{0}, w(x, y, t), \psi_{\beta}$ and $(\beta=x, y)$ are, respectively, the membrane deflection of the middle surface, the transverse deflection of the structure and the rotation of an element normal to the middle surface.

Knowing the displacement field, it is possible to determine the elements of the shear deformation tensor $\varepsilon_{z \beta}$, $(\beta=x, y)$ in the honeycomb core material $[1,9]$.

The fundamental law of elastic behavior and the fundamental relations of the principles of dynamics for bending lead to the following system [9-11]:

$$
\begin{gathered}
h_{a} k_{1} G_{13}\left(\frac{\partial \psi_{x}}{\partial x}+\frac{\partial^{2} w}{\partial x^{2}}\right)+h_{a} k_{1} G_{23}\left(\frac{\partial \psi_{y}}{\partial y}+\frac{\partial^{2} w}{\partial y^{2}}\right) \\
+p=\rho_{s} \frac{\partial^{2} w}{\partial t^{2}} \\
D_{11} \frac{\partial^{2} \psi_{x}}{\partial x^{2}}+D_{66} \frac{\partial^{2} \psi_{x}}{\partial y^{2}}+\left(D_{12}+D_{66}\right) \frac{\partial^{2} \psi_{y}}{\partial x \partial y} \\
-h_{a} k_{1} G_{13}\left(\psi_{x}+\frac{\partial w}{\partial x}\right)=I_{x y} \frac{\partial^{2} \psi_{x}}{\partial t^{2}} \\
D_{22} \frac{\partial^{2} \psi_{y}}{\partial y^{2}}+D_{66} \frac{\partial^{2} \psi_{y}}{\partial x^{2}}+\left(D_{12}+D_{66}\right) \frac{\partial^{2} \psi_{x}}{\partial x \partial y} \\
-h_{a} k_{1} G_{23}\left(\psi_{y}+\frac{\partial w}{\partial y}\right)=I_{x y} \frac{\partial^{2} \psi_{y}}{\partial t^{2}}
\end{gathered}
$$


where:

- $D_{i j}$, and $G_{i j}$ are, respectively, the bending and the shear coefficients [12],

- $k_{1}$ is a correction factor, $k_{1}=5 / 6$ [13];

$-h_{\alpha}$ represents the thickness of the honeycomb core;

$-\psi_{\beta}$ are the rotations of the normal to the middle surface;

$-w$ designs the transverse deflection of the structure;

$-p$ represents the transverse loading applied to the middle surface;

$-I_{x y}$ is the moment of inertia;

$-\rho_{s}$ is the mass density of the plate and is given by $\rho_{s}=\int_{-\frac{h}{2}}^{\frac{h}{2}} \rho \mathrm{d} z=2 h_{p} \rho_{p}+h_{a} \rho_{a}$ where $\rho_{a}$ and $\rho_{p}$ are, respectively, the mass densities of the core and that of the skin of the laminate, $h_{a}$ and $h_{p}$ are respectively, the thickness of the core and of the skin of the laminate.

\subsection{Determination of the transverse displacement of the plate}

The dynamic response of a rectangular, sandwich plate with symmetric, orthotropic skin laminates subjected to an exciting transverse force, can be obtained by modal decomposition [9-11, 14, 15]:

$$
\left[\begin{array}{l}
w(x, y, t) \\
p(x, y, t)
\end{array}\right]=\sum_{m=1}^{\infty} \sum_{n=1}^{\infty}\left[\begin{array}{l}
q_{m n}(t) g_{m n}^{3}(x, y) \\
p_{m n}(t) g_{m n}^{3}(x, y)
\end{array}\right]
$$

The rotations of the normal are written as:

$$
\psi_{\beta}(x, y, t)=\sum_{m=1}^{\infty} \sum_{n=1}^{\infty} a_{m n}^{\beta}(t) g_{m n}^{\beta}(x, y)
$$

The functions $g_{n m}^{i}(x, y),(i=1,2,3, \ldots)$ are known and should satisfy the boundaries conditions. It remains to determine $\alpha_{n m}^{\beta}(t)$ and $q_{n m}(t)$. As far as $\alpha_{n m}^{\beta}(t)$ are concerned, they are obtained from the constitutive relations of the plates based on the known components of the vertical displacement $q_{n m}(t)$. The involvement of the latter in the potential energy of the external load permits the use of Lagrange's method which is a consequence of Hamilton's principle $[11,16]$.

The Lagrangian is defined as:

$$
L=T-V_{T}
$$

where $T$ and $V_{T}$ are, respectively, the kinetic and the potential energies. Then:

$$
\frac{\mathrm{d}}{\mathrm{d} t} \frac{\partial L}{\partial \dot{q}}-\frac{\partial L}{\partial q}=0
$$

$V_{T}$ and $T$ are given by the following expressions:

$$
V_{T}=U_{d}-\frac{1}{2} \iint_{A} q(x, y, t) w(x, y, t) \mathrm{d} x \mathrm{~d} y
$$

$$
\begin{aligned}
& \text { with: } U_{d}=\frac{1}{2} \iint_{A} \sigma^{T} \varepsilon \mathrm{d} x \mathrm{~d} y=\sum_{m} \sum_{n} K_{m n} q_{m n}^{2} \\
& T=\frac{1}{2} \iint_{A} \rho_{s}\left(\dot{u}^{2}+\dot{v}^{2}+\dot{w}^{2}\right)^{2} \mathrm{~d} x \mathrm{~d} y=\sum_{m} \sum_{n} M_{m n} \dot{q}_{m n}^{2}
\end{aligned}
$$

with: $M_{m n}=\rho_{s} \int_{0}^{a} \int_{0}^{b}\left(g_{m n}^{3}(x, y)\right)^{2} \mathrm{~d} x \mathrm{~d} y$.

The solution of the problem is obtained for a concentrated loading:

$$
p(x, y, t)=F(t) \delta\left(x-x_{0}\right) \delta\left(y-y_{0}\right)
$$

For each mode, the Lagrange's method leads to a second order differential equation of the form:

$$
\ddot{q}_{m n}(t)+\omega_{m n}^{2} q_{m n}(t)=\frac{g_{m n}^{3}\left(x_{0}, y_{0}\right) F(t)}{M_{m n}}
$$

The Lagrange solution of equation (6) is written as:

$$
q_{m n}(t)=\frac{1}{M_{n m} \omega_{n m}} \int_{0}^{t} g_{n m}^{3}\left(x_{0}, y_{0}\right) F(\tau) \sin \omega_{n m}(t-\tau) \mathrm{d} \tau
$$

By substituting equation (7) into equation (3), the response of the plate obtained by modal superposition leads to the transverse deflection:

$$
\begin{aligned}
w=\sum_{m=0}^{\infty} \sum_{n=0}^{\infty} \frac{g_{m n}^{3}(x, y) g_{m n}^{3}\left(x_{0}, x_{0}\right)}{M_{m n} \omega_{n m}} \\
\quad \times \int_{0}^{t} F(\tau) \sin \omega_{n m}(t-\tau) \mathrm{d} \tau
\end{aligned}
$$

The natural frequencies $\omega_{m n}$ appeared in equation (8) can be determined experimentally or calculated from the fundamental equations of the sandwiched plate [9].

\subsection{Modal accelerations method}

The modal accelerations method enables one to calculate a statically exact response to the problem of shock loading. Thus, consider the differential equation of an undamped structure subjected to a shock loading based on the Geradin and Rixen model [16]:

$$
M \ddot{U}(t)+K U(t)=F(t)
$$

By truncating the modal series representation of the inertia forces, we can write:

$$
K U(t)=F(t)-\sum_{k=1}^{n} \ddot{q}(t) M \phi_{k}
$$

where $\phi_{k}$ are the modal vectors associated with the pulses $\omega_{k}$. The displacements are then obtained as:

$$
U(t)=K^{-1} F(t)-\sum_{k=1}^{n} \ddot{q}_{k}(t) \frac{\phi_{k}}{\omega_{k}^{2}}
$$


Every modal displacement $q_{k}$ is a solution of a single degree-of-freedom differential equation:

$$
\ddot{q}_{k}(t)+\omega_{k}^{2} q_{k}(t)=\frac{1}{\mu_{k}} \phi_{k}^{T} F(t)
$$

where $\mu_{k}$ is the modal mass $\mu_{k}=\phi_{k}^{T} M \phi_{k}$.

The solution of the system initially at rest is:

$$
q_{k}=\frac{1}{\omega_{k}} \int_{0}^{t} F(\tau) \sin \omega_{k}(t-\tau) \mathrm{d} \tau
$$

Combining equation (12) and equation (13), thus:

$$
\ddot{q}_{k}(t)=\frac{1}{\mu_{k}} \phi_{k}^{T}\left(F(t)-\omega_{k} \cdot \int_{0}^{t} F(\tau) \sin \omega_{k}(t-\tau) \mathrm{d} \tau\right)
$$

Then substituting equation (14) into equation (11), $U(t)$ becomes:

$$
\begin{array}{r}
U(t)=\sum_{k=1}^{n} \frac{\phi_{k} \phi_{k}^{T}}{\omega_{k} \mu_{k}} \int_{0}^{t} \int_{0}^{t} F(\tau) \\
\sin \left(\omega_{k}(t-\tau)\right) \mathrm{d} \tau \\
+\sum_{k=1+n}^{\infty} \frac{\phi_{k} \phi_{k}^{T}}{\omega_{k}^{2} \mu_{k}} F(t)
\end{array}
$$

Finally, the method of modal accelerations resides in completing the response of the modal displacements by including the missing terms in the spectral development of the quasi-static response. In many cases, it can lead to an improvement of the solution without an appreciable increase in computational volume in comparison with the method of modal displacements.

\subsection{Case of a concentrated load}

In order to simulate the experiments dealing with shock loading using drop-weight machines, the excitation is assumed to be vertical, concentrated and directed toward the center of the plate with coordinates $\left(x_{0}, y_{0}\right)$.

The applied loading $p(x, y, t)$ is expanded into a double Fourier series $[9,10,17]$ as:

$$
p(x, y, t)=\sum_{1}^{\infty} \sum_{1}^{\infty} p_{m n}(t) \sin \left(m \pi \frac{x}{a}\right) \sin \left(n \pi \frac{y}{b}\right)
$$

where:

$$
p_{m n}=\frac{4}{a b} \int_{0}^{a} \int_{0}^{b} p(x, y, t) \sin \left(m \pi \frac{x}{a}\right) \sin \left(n \pi \frac{y}{b}\right) \mathrm{d} x \mathrm{~d} y
$$

From equations (16) and (17), the concentrated load becomes:

$$
\begin{array}{r}
p(x, y, t)=\sum_{1}^{\infty} \sum_{1}^{\infty} \frac{4 F(t)}{a b} \\
\sin \left(m \pi \frac{x_{0}}{a}\right) \sin \left(n \pi \frac{y_{0}}{b}\right) \\
\times \sin \left(m \pi \frac{x}{a}\right) \sin \left(n \pi \frac{y}{b}\right)
\end{array}
$$
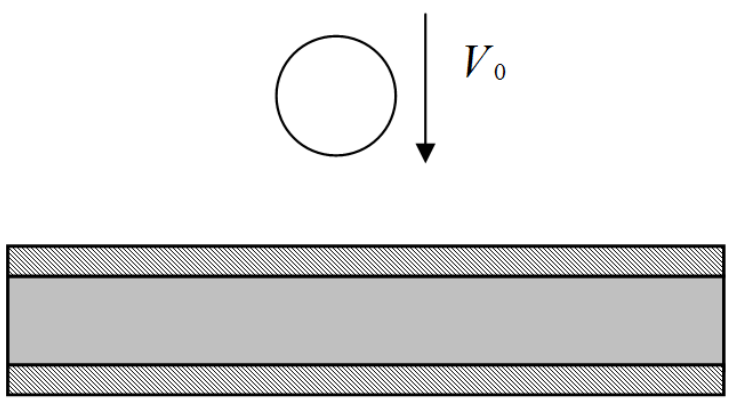

\section{Elastic sandwich plate}

Fig. 1. Configuration example of a falling weight.

\section{Method of solution}

Once it is decided how the plate behaves, how the contact plate-impactor occurs, and how the dynamical phenomenon is discretized, it would be appropriate to explicitly solve the transitional movement of the plate during and after the impact.

\subsection{Contact force}

Consider two bodies, one elastic and one rigid, which are about to collide as shown in Figure 1.

Within the structure of the study of the behavior of a sandwiched plate subjected to loading shock, this contact can be modeled following the Hertz's law [6,17-19]. Thus, the contact force of a sphere on a plane surface is of the type:

$$
F=k_{\mathrm{h}}(\alpha)^{3 / 2}
$$

where $F$ is the contact force during the shock loading, $k_{\mathrm{h}}$ is the Hertzian coefficient which depends on the elastic properties of both bodies. This coefficient can be determined experimentally by quasi-static indentation $[18,20-22]$. The penetration is given by:

$$
\alpha=s(t)-w\left(x_{0}, y_{0}, t\right) \quad \text { for } \quad t>0
$$

where $w\left(x_{0}, y_{0}, t\right)$ is the transverse displacement of the structure at the point of impact and $s(t)$ is the displacement of the impactor.

\subsection{Impactor displacement}

To calculate the displacement of the impactor having a mass equal to $m_{0}$, the fundamental principle of dynamics is applied to the falling weight. Thus:

$$
-F=m_{0} \frac{\mathrm{d} v}{\mathrm{~d} t}
$$


where $F$ is the contact force (determined as being an external action on the plate) at time $t$.

The variation of the velocity during $\mathrm{d} \tau$ is expressed as follows:

$$
\mathrm{d} V=-\frac{F(\tau)}{m_{0}} \mathrm{~d} \tau
$$

The velocity of the impactor at time $t$ is determined by integration. Therefore:

$$
V(t)=V_{0}-\frac{1}{m_{0}} \int_{0}^{t} F(\tau)(t-\tau) \mathrm{d} \tau
$$

where $V_{0}$ is the instantaneous velocity before the shock. It can be determined from the conservation of energy theorem expressed by:

$$
\frac{1}{2} m_{0} V_{0}^{2}=m_{0} g z_{0}
$$

This gives:

$$
V_{0}=\sqrt{2 g z_{0}}
$$

where $z_{0}$ is the height drop.

The variation of the displacement during $(t-\tau)$ is equal to:

$$
\mathrm{d} s(t)=\mathrm{d} V(t-r)
$$

Finally, the transverse displacement of the spherical impactor of mass $m_{0}$ at any time is obtained by a new integration:

$$
s(t)=V_{0} t-\frac{1}{m_{0}} \int_{0}^{t} F(\tau)(t-\tau) \mathrm{d} \tau
$$

The penetration law, according to the theory of Hertz, is expressed as:

$$
\alpha=s(t)-w\left(x_{0}, y_{0}, t\right)
$$

Then, the transverse displacement of the structure is expressed as:

$$
\begin{aligned}
w(x, y, t)= & \sum_{1}^{N} \sum_{1}^{N} \frac{g_{m n}^{3}(x, y) g_{m n}^{3}\left(x_{0}, y_{0}\right)}{M \omega_{n m}} \\
& \times \int_{0}^{t} F(\tau) \sin \omega_{m n}(t-\tau) \mathrm{d} \tau \\
& +\sum_{N+1}^{\infty} \sum_{N+1}^{\infty} 4 \frac{g_{m n}^{3}(x, y) g_{m n}^{3}\left(x_{0}, y_{0}\right)}{a b \rho_{s} \omega_{m n}^{2}} F(t)
\end{aligned}
$$

Substituting equations (24)-(26) into equation (20), a nonlinear integral equation that can only be solved numerically is obtained. Thus:

$$
\begin{gathered}
\left(F(t) / k_{\mathrm{h}}\right)^{2 / 3}=V_{0} t-\frac{1}{m_{0}} \int_{0}^{t} F(\tau)(t-\tau) \mathrm{d} \tau \\
-\sum_{1}^{N} \sum_{1}^{N} \frac{g_{n m}^{3}(x, y) g_{n m}^{3}\left(x_{0}, y_{0}\right)}{M \omega_{n m}} \int_{0}^{t} F(\tau) \sin \omega_{n m}(t-\tau) \mathrm{d} \tau \\
-\sum_{N+1}^{\infty} \sum_{N+1}^{\infty} 4 \frac{g_{m n}^{3}(x, y) g_{m n}^{3}\left(x_{0}, y_{0}\right)}{a b \rho_{s} \omega_{m n}^{2}} F(t)
\end{gathered}
$$

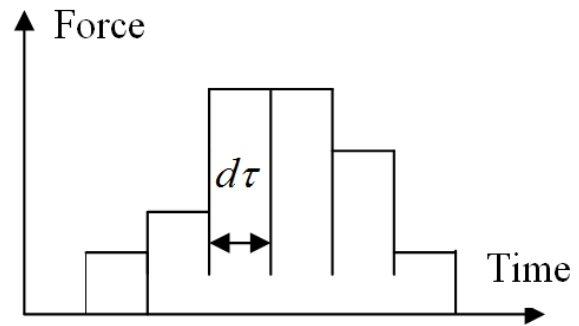

Fig. 2. Numerical schematic of integration.

To calculate the contact force, an iterative method based on a numerical scheme for explicit time integration is used (Fig. 2). This is an extension of a method in which the contact force is assumed constant during the interval $[i \mathrm{~d} \tau$, $(i+1) \mathrm{d} \tau]$.

\subsection{Iterative method}

The contact force during a small time interval is assumed to be constant. Initially, for $t=0$ and at a first time increment, $F_{1}$ is calculated from $V_{0}$, thus:

$$
F_{1}=k_{h}\left(V_{0} \mathrm{~d} \tau\right)
$$

At an increment time $i, F_{i}$ is calculated from $V_{(i-1)}$ and $F_{(i-1)}$, thus:

$$
\begin{aligned}
\left(F_{i} / k_{\mathrm{h}}\right)^{2 / 3}= & V_{0}(i-1) \mathrm{d} \tau-\frac{1}{m_{0}} \mathrm{~d} \tau^{2} \sum_{j=1}^{i} F_{j} \frac{(2 i-2 j-1)}{2} \\
& -\sum_{n}^{\infty} \sum_{m}^{\infty} \frac{\left(g_{n m}^{3}\left(x_{0}, \quad y_{0}\right)\right)^{2}}{M_{m n} \omega_{m n}} \sum_{j=0}^{i-1} F_{j}\left(\cos \omega_{m n}\right. \\
& \left.\times(i-j-1) \mathrm{d} \tau-\cos \omega_{m n}(i-j) \mathrm{d} \tau\right) \\
& -\sum_{n}^{\infty} \sum_{m}^{\infty} \frac{\left(g_{m n}^{3}\left(x_{0}, y_{0}\right)\right)^{2}}{M_{m n} \omega_{m n}^{2}} F_{i-1}
\end{aligned}
$$

The iterations are continued until a number $N$ is obtained such that $F_{N}=0$. Thus, $N \mathrm{~d} \tau$ is the contact time.

\section{Comparison trial/calculation}

The calculation of the deformations during the time the shock is applied has been performed on rectangular sandwiched plate $\left(300 \times 300 \times 24 \mathrm{~mm}^{3}\right)$, simply supported all around $\left(300 \times 300 \mathrm{~mm}^{2}\right.$ of free surface), containing two identical skin layers made of fibers glass "rovimat" and $2 \mathrm{~mm}$ polyester in thickness, and of foamy core material PVC2. This plate is subjected to an impacting rigid sphere falling from a height varying between $1 \mathrm{~m}$ and $2.5 \mathrm{~m}$. The impactor that is used has a mass of $10.9 \mathrm{~kg}$ and a radius of $50 \mathrm{~mm}$. The geometric characteristics of the plate and its material properties are shown in Table 1. 
Table 1. The used plate characteristics.

\begin{tabular}{ccc}
\hline Property & Skin (laminated) & Core \\
\hline Mass density kg.m & 1630 & 80 \\
Young's modulus & $E_{1}=E_{2}=14 \mathrm{GPa}$ & $E_{\mathrm{pvc} 2}=56 \mathrm{MPa}$ \\
Shear modulus of elasticity & $G_{12}=4 \mathrm{GPa}$ & $G_{\mathrm{pvc} 2}=20 \mathrm{MPa}$ \\
Poisson's ratio & $v_{12}=0.25$ & $v_{12}=0.4$ \\
Thickness (mm) & 2.2 & 20 \\
\hline
\end{tabular}

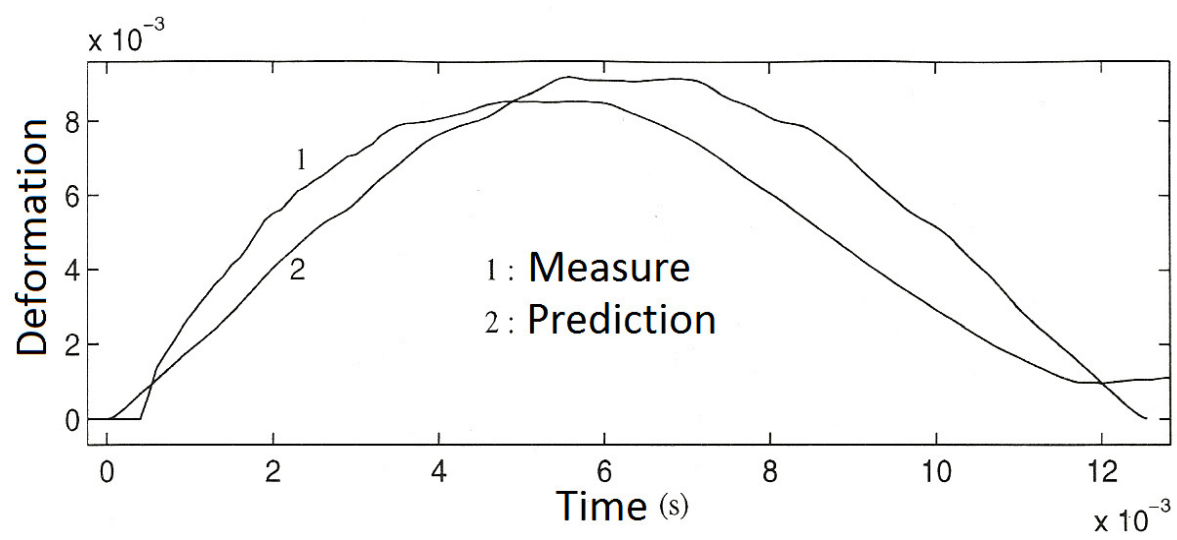

Fig. 3. Deformation for $10.9 \mathrm{~kg}$ and $2 \mathrm{~m}$ of height.

Table 2. Deformations (predicted/measured) at the PVC2 sandwiched plate center.

\begin{tabular}{ccc}
\hline Height $(\mathrm{m})$ & $\varepsilon_{x x}\left(10^{-3}\right)$ & $\varepsilon_{y y}\left(10^{-3}\right)$ \\
\hline 1 & $6.5 / 7.1$ & $6.00 / 6.45$ \\
& $-9.2 \%$ & $-7.5 \%$ \\
1.5 & $8.7 / 8.4$ & $8.78 / 8.2$ \\
& $3.4 \%$ & $6.6 \%$ \\
2 & $8.95 / 8.38$ & $10.2 / 9.41$ \\
& $6.3 \%$ & $7.7 \%$ \\
2.5 & $11.4 / 8.13$ & $11.4 / 9.77$ \\
& $28.6 \%$ & $14.29 \%$ \\
\hline
\end{tabular}

The plate length is: $a=0.3 \mathrm{~m}$. Its width is: $b=0.3 \mathrm{~m}$. The coefficient of penetration, obtained through a quasistatic indentation, is: $k_{\mathrm{h}}=2 \times 10^{7} \mathrm{~N} \cdot \mathrm{m}^{-1.5}$.

The validation at the deformations level is also conducted in the case of a simply supported sandwich plate. The results of measurements at the center of the underside are illustrated in Figure 3 and are compared with results from the analytical model. Relative deviations are observed between the measured and calculated values in Table 2. The difference between the two results increases more and changes from negative to positive, this can be explained by the fact that the strain rate affects the stiffness of the skins as well as the Young's modulus. This has been observed for PVC2 materials for which the influence of speed is very important.

Other comparisons have been made (for the same type of sandwiched plates [23,24]). They also show a good correlation between experiment and simulation. Given the discrepancies existing in the material properties and the errors in measurements, the proposed method does allow the prediction of the behavior of the plate subsequent to impact.

\section{Parametric study}

Table 3 shows that some characteristics of the response of the plate (sandwich panel) are affected by the material properties.

It can be noted that the Hertz factor $k_{\mathrm{h}}$ does not have a strong influence. On the other hand, the contact forces, the displacements and the flexural deflections are affected by the thickness of the skin layers, and, to a lesser extent, by the thickness and the shear modulus of elasticity $G_{13}$ of the core. However, the shear, which is a determining factor for damage prediction, is principally influenced by both the thickness of the core and its modulus of elasticity $G_{13}$.

\section{Conclusion}

The objective of this work is to develop an analytical approach using modal acceleration, based on the classical theory of sandwiched, laminated plates, to predict the occurrence of global damage by first considering the static solution.

By using the Hertz's law of indentation, the model is used to analyze the dynamical behavior of such plates by calculating the contact force, the deflection, and the strains occurring at the lower skin layer, at the center of the plate during impact. The given results from the presented model have been compared to results generated 
Table 3. Influence of the material parameters on the dynamical response.

\begin{tabular}{cccccc}
\hline Parameter & Force 1 & Deflection 2 & $\varepsilon_{x x}^{(1)}$ & $\varepsilon_{x z}^{(2)}$ & Duration of impact \\
\hline$k_{\mathrm{h}}+10 \%$ & $1.4 \%$ & $1.7 \%$ & $0 \%$ & $1 \%$ & $-0.7 \%$ \\
$E_{i}+10 \%$ & $1.0 \%$ & $-1.0 \%$ & $-7 \%$ & $1 \%$ & $0.0 \%$ \\
$G_{13}+10 \%$ & $4.0 \%$ & $-4.0 \%$ & $3 \%$ & $-5 \%$ & $-9.0 \%$ \\
$h_{a}+10 \%$ & $10.0 \%$ & $-7.0 \%$ & $-6 \%$ & $-6 \%$ & $-4.0 \%$ \\
$h_{p}+10 \%$ & $1.0 \%$ & $0.0 \%$ & $-9 \%$ & $0 \%$ & $0.0 \%$ \\
\hline
\end{tabular}

from experiments. Thus, experimental results validate the proposed model.

The study of affecting material parameters shows that the latter have an influence on the dynamical behavior of the sandwiched panel. In addition, this study provides a basis to develop an experimental procedure to analyze these parameters. However, it should be noted that the approach used in this work, specifically, to vary the parameters individually, is somewhat simple. It should be noted that this approach does not take into consideration the numerous relationships existing between these parameters. For example, the shear modulus of elasticity $G_{13}$ has an influence on both the response of the panel and on the coefficient of indentation.

\section{References}

[1] R.D. Mindlin, Influence of rotatory inertia shear on flexural motions of isotropic elastic plates, J. Appl. Mech. 18 (1951) 31-38

[2] J.M. Whitney, N.J. Pagano, Shear Deformation in Heterogeneous Anisotropic Plates, J. Appl. Mech. (1970) $1031-1036$

[3] S.P. Timoshenko, J.N. Goodier, Theory of elasticity, McGraw-Hill, New York, 1934

[4] W. Goldsmith, Impact: the Theory and Physical Behavior of Colliding Solids, Edward Arnold Ltd, London Publishers, 1960

[5] C.T. Sun, S. Chattopadhyay, Dynamic Response of Anisotropic Laminated Plates Under Initial Stress to Impact of Mass, J. Appl. Mech. 42 (1975) 693-698

[6] A.L. Dobyns, Analysis of simply-supported orthotropic plates subject to static and dynamic loads, AIAA J. 19 (1981) 642-650

[7] J.K. Chen, C.T. Sun, Analysis of impact response of buckled composite laminates, Compos. Struct. 3 (1985) 97-118

[8] C. Doan, P. Hamelin, Comportement au choc des matériaux et structures composites, Caractérisation mécanique des composites, Pluralis, Vautrin Ed, 1989, pp. $157-176$

[9] J.M. Whitney, Structural Analysis of Laminated Anisotropic Plates, Technomic Publishing Co., 1987

[10] R.M. Hussein, Composite panels/plates analysis and design, Technomic Publishing Co., 1986

[11] A.P. Christoforou, S.R. Swanson, Analysis of impact response in composite plates, Int. J. Solids Struct. 27 (1991) $161-170$
[12] J.M. Berthelot, Matériaux Composites: Comportement mécanique et analyse des structures, Edition Masson, 1992

[13] J.M. Whitney, A.W. Leissa, Analysis of a simply supported laminated anisotropic rectangular plates, AIAA J. 8 (1970) 28-33

[14] G. Cederbaum, I. Elishakoff, J. Aboudi, L. Liberscu, Random Vibration and Reliability of Composite Structures, Technomic Publishing Co., 1992

[15] A.S. Yigit, A.P. Christoforou, On the Impact of a Spherical Indenter and an Elastic-plastic Transversely Isotropic Half-Space, Compos. Eng. 4 (1994) 1143-1573

[16] M. Geradin, D. Rixen, Théorie des vibrations, Masson, Paris, 1993

[17] S. Thangjitham, L. Librescu, G. Cederbaum, LowVelocity Impact Response of Orthotropic Plates Using a Higher-Order Theory, Proceedings of the 28th AIAA/ASME/ASCE/AHS: Structures, Structural Dynamics and Materials Conference, Monterey, California, 1987, pp. 448-457

[18] O.T. Thomsen, Theoretical and experimental investigation of local bending effects in sandwich plates, Compos. Struct. 30 (1995) 85-101

[19] B.V. Sankar, Low-Velocity Impact Response and Damage in: E. Armanios (Ed.), Composite Materials, Fracture of Composites, 1996

[20] T.M. Tan, C.T. Sun, Use of statical indentation Laws in the impact analysis of laminated composite plates, J. Appl. Mech. 52 (1985) 6-12

[21] R.A. Mines, C.M. Worral, A.G. Gibson, The response of GRP sandwich panels to dropped object loading, FRC'90, 4th International Conference on Fiber Reinforced Composites, Liverpool, United Kingdom, 1990, pp. 149-155

[22] P. Davies, D. Choqueuse, L. Riou, A. Wahab, Réponse de panneaux composites sandwichs au choc, Composite Materials in the Petroleum Industry, Paris, France, 1994, IFP Revue, 1995, pp. 1-8

[23] P. Davies, D. Choqueuse, B. Bigourdan, Static and sandwich impact testing and modelling of sandwich structures for marine applications, 3rd International Conference on Sandwich Construction, Southampton, United Kingdom, 1995, pp. 647-658

[24] A. Wahab, N. Moubayed, P. Davies, M. Prevosto, Y. H. De Roeck, Simulation de la réponse dynamique d'une poutre sandwich en matériaux composites à l'impact d'une sphère rigide, Matériaux et Techniques, EDP Sciences 98 (2010) 339-346 